\title{
Epidermal CFTR Suppresses MAPK/NF-KB to Promote Cutaneous Wound Healing
}

\author{
Jing Chen ${ }^{\mathrm{a}}$ Yu Chen ${ }^{\mathrm{a}}$ Yajie Chen ${ }^{\mathrm{a}}$ Zicheng Yang ${ }^{\mathrm{a}}$ Bo You ${ }^{\mathrm{a}}$ Ye Chun Ruan ${ }^{\mathrm{b}}$ \\ Yizhi Peng ${ }^{\mathrm{a}}$ \\ aState Key Laboratory of Trauma, Burns and Combined Injury, Chongqing Key Laboratory for \\ Proteomics Disease, Institute of Burn Research, Southwest Hospital, the Third Military Medical \\ University, Chongqing, China, ${ }^{b} E$ pithelial Cell Biology Research Centre, School of Biomedical Sciences, \\ Faculty of Medicine, the Chinese University of Hong Kong, Hong Kong
}

\author{
Key Words \\ CFTR • Cutaneous wound healing $• \mathrm{MAPK} \cdot \mathrm{NF}-\mathrm{KB}$
}

\begin{abstract}
Background: CFTR is implicated in cutaneous wound healing although the underlying mechanisms are not fully understood. In other cell types, CFTR is reported to regulate MAPK/ NF-KB signaling. We undertook the present study to explore a possible role of CFTR in regulating MAPK/NF-KB during cutaneous wound healing. Methods\& Results: The splintexcisional and incisional wound healing models were used in CFTR mutant (DF508) mice. The cell-scratch model was used in a human keratinocyte line, $\mathrm{HaCaT}$, in conjunction with CFTR knockdown or overexpression. The epidermal inflammation, keratinocyte proliferation and differentiation, as well as MAPK/NF- $\mathrm{kB}$ signaling were examined. Inhibitors of MAPK/NF-KB were also used. Results: Both DF508 mice and HaCaT cells with CFTR knockdown exhibited delayed cutaneous wound healing with exuberant inflammation, increased proliferation and aberrant differentiation. Knockdown of CFTR in HaCaT cells resulted in phosphorylation of ERK, p38 and IKB $\alpha$. The disturbance of inflammation, proliferation and differentiation in $\mathrm{HaCaT}$ cells were reversed by CFTR overexpression or inhibition of MAPK or NF- $\mathrm{kB}$. Conclusion: CFTR plays a role in suppressing MAPK/NF-KB to relieve inflammation, reduce proliferation and promote differentiation of keratinocytes, and thus promotes cutaneous wound healing.
\end{abstract}

\section{Introduction}

Published by S. Karger AG, Basel

Cutaneous wound healing is a highly orchestrated and complex process involving multiple types of cells in the epidermis and dermis [1,2]. Among them, the keratinocyte, a major cellular component of the epidermis, plays an important role in inflammation, proliferation and differentiation during epidermis repair after injuries [1]. A broad variety of molecules and signaling pathways are identified to be important to cutaneous wound healing

J. Chen and Y. Chen contributed equally to this work.

Yizhi Peng

and Ye Chun Ruan

KARGER
State Key Laboratory of Trauma, Burns and Combined Injury, Chongqing Key Laboratory for Proteomics Disease, Institute of Burn Research, Southwest Hospital, the Third Military Medical University, Chongqing (China) and Epithelial Cell Biology Research Centre, School of Biomedical Sciences, Faculty of Medicine, the Chinese University of Hong Kong, (Hong Kong), E-Mail yizhipen@sina.com / ruanyechun@cuhk.edu.hk 


\section{Cellular Physiology Cell Physiol Biochem 2016;39:2262-2274

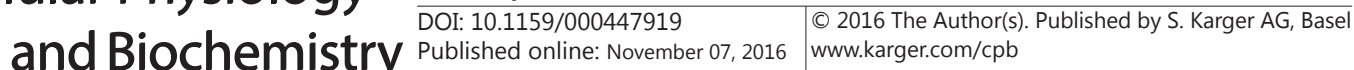 \\ Chen et al.: CFTR Suppresses MAPK/NF-kB in Cutaneous Wound Healing}

[3-5]. Nuclear factor kappa-light-chain-enhancer of activated B cells (NF- $\kappa$ B) and mitogenactivated protein kinase (MAPK), two key signaling pathways involved in inflammation [6, 7], cell proliferation [8, 9] and differentiation [8-14] in many cell types and tissues, have also been implicated in cutaneous wound healing $[3,4]$. However, the molecular mechanisms underlying cutaneous wound healing remain unclear.

Cystic fibrosis transmembrane conductance regulator (CFTR), is a cAMP-dependent anion channel, mutations in which cause cystic fibrosis (CF), an autosomal recessive disease $[15,16]$. Of the identified 1,900 mutations of CFTR in humans, deletion of the phenylalanine at position 508 (DF508) is the most frequent mutation found in CF patients, which results in a folding defect and endoplasmic reticulum associated degradation of CFTR [17]. It is being recently recognized that apart from transporting anions, CFTR is involved in other cellular functions, including inflammation [6, 18], proliferation [19-21], and differentiation [22-24] in bronchial epithelia, granulosa cells, enterocytes, and osteoblasts. Several signaling molecules associated with CFTR have been reported, including MAPK and NF- $\mathrm{B}[6,18,25-28]$. We have previously demonstrated that in the airway, CFTR plays a role in suppressing MAPK/ $\mathrm{NF}-\kappa \mathrm{B}$ signaling in pulmonary inflammation induced by thermal inhalation injury[29].

Interestingly, the expression of CFTR in the epidermis in both humans [30] and mice [31] have been reported. In a recent study, CFTR has been demonstrated to promote the formation of cellular-junctions in human keratinocytes and thus be beneficial for wound repair [31]. Using an excisional wound healing model, the same study has also shown that mice carrying DF508 mutation had delayed wound healing as compared to the wildtypes [31]. However, whether and how CFTR is involved in inflammatory or cell proliferation signaling(s) during wound healing remain elusive. Given the observed role of CFTR in regulation of MAPK/NF$\kappa \mathrm{B}$-mediated inflammation in other systems, we hypothesized that epidermal CFTR may also play a role in epidermal inflammation, proliferation and differentiation during cutaneous wound healing by regulating MAPK/NF- $\mathrm{BB}$ signaling pathway. In the present study, DF508 mutant mice were also used to assess their wound repair. Different from the previous study [31], we used a splinting excisional wound healing model, in which wound contractions are restrained to better mimic wound healing in humans [32]. In addition, we also used an incision wound model in DF508 mice as well as a human epidermal keratinocyte cell line (HaCaT) in conjunction with knockdown or overexpression of CFTR.

\section{Materials and Methods}

Animals and procedures

The $c f t r^{\mathrm{tm} 1 \mathrm{Kth}}$ (DF508) mice were purchased from the Jackson Laboratory [31] and kept at the animal facility of the Third Military Medical University. Male adult DF508 mice were used for cutaneous wound healing models. All procedures were approved by the Animal Ethical Committee of the Third Military Medical University and were carried out in accordance with the approved guidelines. Mice were anesthetized, shaved at the back and sterilized with $70 \%$ alcohol. Excisional full-thickness skin wounds were made on the dorsal skin using a sterile disposable biopsy punch with a diameter of $5 \mathrm{~mm}$. The wounds were immediately covered with a biological membrane as splint to restrain wound contractions. In another set of experiments, two full thickness incisional wounds $(1 \mathrm{~cm}$ long each) were cut in parallel with equal distance $(0.5 \mathrm{~cm})$ to the midline. Animals were individually caged to avoid possible traumatic damage to the wounds. Pictures were taken every day after the surgical injuries with a piece round paper ( $5 \mathrm{~mm}$ diameter) placed next to the wound as a size reference. The size of the wounds was measured by Adobe Photoshop software to calculate wound closure rate.

\section{Cell culture}

HaCaT cells were grown in Calcium-free RPMI-1640 medium (HYCLONE, Cat\#SH30809.01) with $10 \%$ fetal bovine serum in $\mathrm{CO}_{2}(5 \%)$ incubator at $37^{\circ} \mathrm{C}$ [33]. To induce differentiation, $\mathrm{CaCl}_{2}(1 \mathrm{mM})$ was supplemented into the culture medium to incubate HaCaT cells for 48 hours. Primary mouse keratinocytes were isolated and cultured as previously described [34]. In some experiments, Bay-11 (Cat\#B5556, Sigma, 


\section{Cellular Physiology Cell Physiol Biochem 2016;39:2262-2274

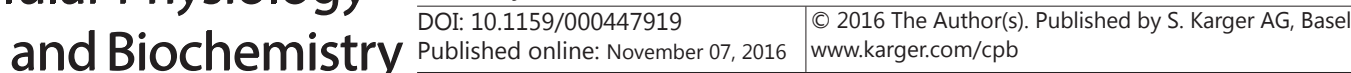 \\ Chen et al.: CFTR Suppresses MAPK/NF-kB in Cutaneous Wound Healing}

ST. Louis, USA), PD98059 (Cat\#P215, Sigma) or SB203580 (Cat\#S8307, Sigma) were added to the culture media.

\section{Cell scratch model}

HaCaT cells were plated in 24-well plates and grown till confluence. A line gap, mimicking an incisional wound in vivo, was made at the center of each well by scratching using a sterile $200 \mu \mathrm{l}$ pipette tip. Afterwards, cells were washed with PBS for three times before fresh growth medium was added. Pictures of cells were taken by phase-contrast microscopy (OLYMPUS, Japan) every hour after the scratch for 20-25 hours. NIH ImageJ image software was used for analysis.

\section{CFTR knock-down and overexpression}

CFTR was knocked down using hammerhead ribozymes (conjugated in pEF6/V5-His vector) targeting at a specific GUC or AUC site to degrade CFTR mRNA as described previously [29]. To overexpress CFTR, pEGFPC3 plasmid carrying wild-type human CFTR (a gift from Professor Tzyh-Chang Hwang, University of Missouri-Columbia, USA) was used. Empty pEF6/V5-His or pEGFPC3 vectors was used as controls for knockdown or overexpression experiments, respectively. Cells were transfected with $4 \mu \mathrm{g}$ plasmids using lipofectamine 2000 reagent (Invitrogen) according to the manufacturer's instructions.

Quantitative real-time RT-PCR (QRT-PCR)

QRT-PCR was carried out using a cDNA Synthesis Kit (Cat\#K1622, Thermo Scientific, Fremont, USA) and the SGExcelUltraSYBR Mixture (Cat\#SK2956A, Sangon Biotech, Shanghai, China). $\triangle \triangle \mathrm{Ct}$ was calculated and statistically analyzed. The sequences of primers used were as follows: CFTR: TGCCCTTCGGCGATGTTT (forward), GCGATAGAGCGTTC CTCCTTG (reverse); COX-2: TGTGTTGACATCCAGATCAC (forward), ACATCATGTTTGAGCCCTGG (reverse); $\beta$-actin: CATGTACGTTGCTATCCAGGC (forward), CTCCTTAATGTCACGCACGAT (reverse); IL-8: GGTGGAGTTTGCCAAGGAG (forward), TTCCTTGGGGTCCAGACAGA (reverse). IL-10: GACTTTAAGGGTTACCTGGGTTG (forward), TACCATGCGCCTTGATGTCTG (reverse); CALM5: GGTTGACACGGATGGAAACG (forward), ACTCCTGGAAGCTGATTTCGC (reverse); LOR: GCTCTCATGATGCTACCCGA (forward), TGGGGTTGGGAGGTAGTTGT (reverse); IVL: TCCTCCAGTCAATACCCATCAG (forward), CAGCAGTCATGTGCTTTTCCT (reverse); FLG: TGAAGCCTATGACACCACTGA (forward), TCCCCTACGCTTTCTTGTCCT (reverse).

\section{Western blot}

Western blot was carried out as previously described [29]. Antibodies used in this study were listed as follows: CFTR (1:200, Cat\#ACL-006, Alomone labs), PCNA (1:1000, Cat\#D120014, BBI life Sciences), FLG (1:1000, Cat\#ab24584, Abcam Inc.), LOR (1:1000, Cat\#ab24722, Abcam Inc.), IVL (1:1000, Cat\#ab53112, Abcam Inc.), P-Erk1/2 (1:1000, Cat\#4370, Cell Signaling Technology), Erk1/2 (1:1000, Cat\#4695, Cell Signaling Technology), P-p38 MAPK (1:1000, Cat\#4511, Cell Signaling Technology), p38 MAPK (1:1000, Cat\#9212, Cell Signaling Technology), P-IкB $\alpha$ (1:1000, Cat\#2859, Cell Signaling Technology), IкB $\alpha$ (1:1000, Cat\#4814, Cell Signaling Technology), Tubulin (1:500, Cat\#10759-1-AP, Proteintech, Chicago, USA) and $\beta$ -actin (1:1000, Cat\#60008-1-lg, Proteintech).

\section{Histological analysis}

Tissues were fixed in 4\% PFA, paraffin-embedded and sectioned. A hematoxylin and eosin (H\&E) kit was purchased from Sangon Biotech (Cat\#E607318-0200). Experiments were performed according to manufacturer's instructions. Histological wound width was quantified by measuring the distance between the leading edges of epidermis across the wound bed. Relative inflammatory infiltration in 8 animals per genotype ( 2 randomly chosen wound edge fields and 2 randomly chosen wound bed fields per animal) was semi-quantitatively evaluated by three blinded reviewers. Epithelial thickness was quantified by using the measure function of the Adobe Photoshop software.

Immunohistochemistry

For immunohistochemistry, after antigen retrieval, sections were stained overnight at $4{ }^{\circ} \mathrm{C}$ with PCNA (1:100 diluted, BBI life Sciences). After washing, sections were stained with the secondary antibodies with peroxidase-conjugated anti-rabbit followed by rabbit peroxidase anti-peroxidase (Dako 


\section{Cellular Physiology Cell Physiol Biochem 2016;39:2262-2274

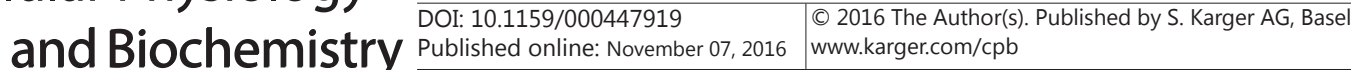 \\ Chen et al.: CFTR Suppresses MAPK/NF-kB in Cutaneous Wound Healing}

Corporation, Carpinteria, CA) for $1 \mathrm{~h}$ at room temperature. After being washed, color was developed with 3,3-diaminobenzidine and counterstained with hematoxylin. Sections with the primary antibody omitted were used as a negative control.

\section{Proliferation assay}

Cells were plated in a 96-well plate at a density of $1 \times 10^{4}$ cells per well and a cell counting kit (SK6335500T, BBI Life Science) was used to determine the proliferation rate of cells. Absorbance was measured at 450nm using an ELx800 Absorbance Microplate Reader (Bio-Tek Instruments, Winooski, VT).

$P G E_{2}$ and IL-8 ELISA

ELISA kits for human PGE $_{2}$ (ESK5090-96 T) and IL-8 (ESK5204-96 T) were purchased from Sangon Biotech and used according to manufacturer's instructions.

Statistical analysis

Data are the mean \pm sem. Students'-test was used when comparing two groups of variables. Two Way or One-way ANOVA followed by post-tests was used for multiple-groups comparisons. Alltests were twotailed, and statistical significance was set at $\mathrm{P}<0.05$.

\section{Results}

Delayed cutaneous wound healing in DF508 mice

We first made excisional wounds on dorsal skin of mice, which were immediately covered by a biological membrane as a "splint" to restrain contractions during the repair as previously reported [32]. In wildtype $(+/+)$ mice, the wounds were gradually closed up by $\sim 90 \%$ within 7 days. With the same injury, the DF508 (-/-) mice showed delayed wound healing with significantly lower wound closure rate at day 3, 5 and 7 (Fig. 1a). For a better histological assessment of wound healing[35], we made incisional skin injury to the DF508 mice (Fig. 1b). H\&E staining showed significant larger wound width (Fig. 1c and d) and lower re-epithelialization rate (Fig. 1e) in DF508 mice at day 3 and 5 post injury as compared to the wildtypes.

\section{CFTR promotes keratinocyte wound healing in vitro}

We next used an in vitro scratch wound healing model in HaCaT cells, an immortalized human keratinocyte line. A line gap in HaCaT cultures was made by a scratch, resembling a "wound", which was gradually filled up with migrating cells. Within 20 hours, the scratched gap was closed by 60-70\%. In presence of a selective CFTR inhibitor (CFTRinh172, 10 $\mu \mathrm{M}$ ), the closure rate at 20 hours was significantly lower than cells treated with DMSO as a vehicle control (Fig. 2a). To confirm this, we knocked down CFTR expression in the cells by transfecting CFTR-targeting ribozymes. QRT-PCR and western blot assay confirmed $\sim 75 \%$ knockdown of CFTR (Fig. 2b). With such knockdown of CFTR, cells showed significantly lower closure rate than control cells (Fig. 2c). To further confirm the involvement of CFTR, we transfected the cells with EGFPC3 plasmid carrying human CFTR, which resulted in robust increase in CFTR expression (Fig. 2d). With such overexpression of CFTR, HaCaT cells showed significantly greater closure rate at 25 hours as compared to control cells (Fig. 2e).

\section{CFTR deficiency enhances epidermal inflammation}

The production and release of cytokines and chemokines by keratinocytes to recruit inflammatory leukocytes (i.e. neutrophils and macrophages) are the early events upon skin injuries, whereas over-inflammation may impair the healing process [36]. Since CFTR has been reported to suppress inflammation in other cell types (such as airway epithelial cells) $[6,37,38]$, we tested whether CFTR is associated with inflammatory responses during skin injury and repair. We first examined leukocytes infiltration in the incisional wound in wildtype $(+/+)$ and DF508 (-/-) mice. At 10 hours, 3 and 5 days after the incisional injury, significantly 


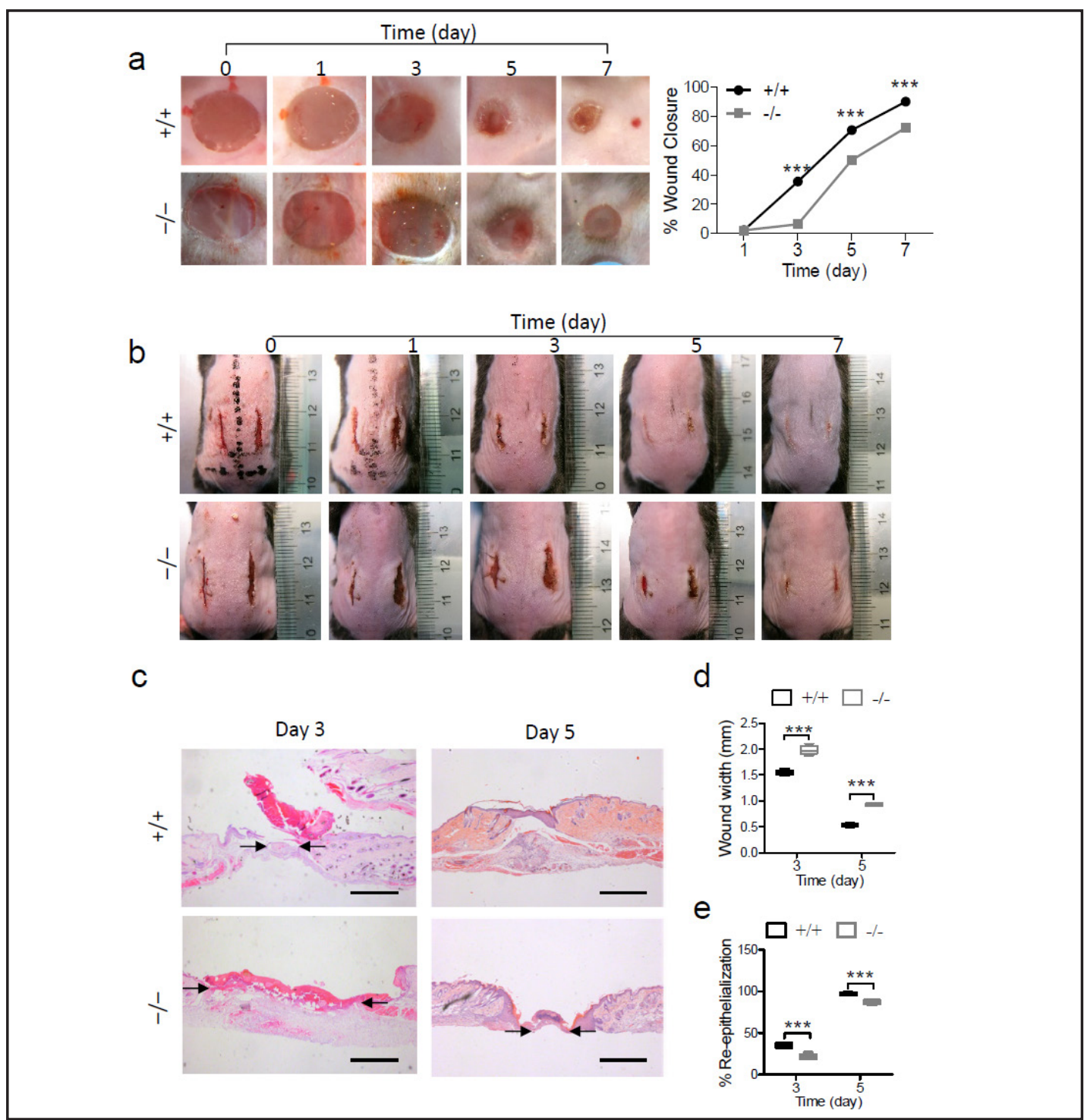

Fig. 1. Delayed cutaneous wound healing in DF508 mice. a) Representative photograph of excisional wound and quantification of wound closure rate in DF508 (-/-) or wildtype (+/+) mice at day 0-7 post-surgical injury. The rate of wound closure was defined as the percentage of closed wound area size versus the initial wound area size. ${ }^{* *}: \mathrm{P}<0.001, \mathrm{n}=6$, Two-way ANOVA. b) Representative photograph of incisional wound of DF508 (-/-) or wildtype (+/+) mice at day 0-7 post injury. c) Hematoxylin and eosin staining of incisional wounds of DF508 (-/-) or wildtype (+/+) mice at day 3 and 5 post injury. The wound edges were marked by arrows. Bar $=200 \mu \mathrm{m}$. d-f) Quantification of incisional wound width (d) re-epithelialization rate (f)in DF508 $(-/-)$ or wildtype $(+/+)$ mice at day 3 and 5 post injury. $* * *: \mathrm{P}<0.001, \mathrm{n}=6$, Two-way ANOVA.

larger number of neutrophils and macrophages in the wound bed were observed in DF508 mice as compared to that in wildtypes (Fig. 3a). In HaCaT cells, we examined the production and release of inflammatory cytokines and chemokines that are associated with cutaneous wound healing. As shown in Fig. 3b-f, knockdown of CFTR led to elevations of COX-2/PGE (Fig. 3b) and IL-8 (Fig. 3d). The effect was found more robust in the presence of TNF $\alpha(10 \mu \mathrm{M})$, a factor increased immediately upon skin injuries [39]. Conversely, overexpression of CFTR significantly reduced COX-2 (Fig. 3c) and IL-8 (Fig. 3e) expression in HaCaT cells. Moreover, IL-10, an anti-inflammatory cytokine, was decreased by CFTR knockdown and increased by its overexpression (Fig. 3f).

\section{KARGER}




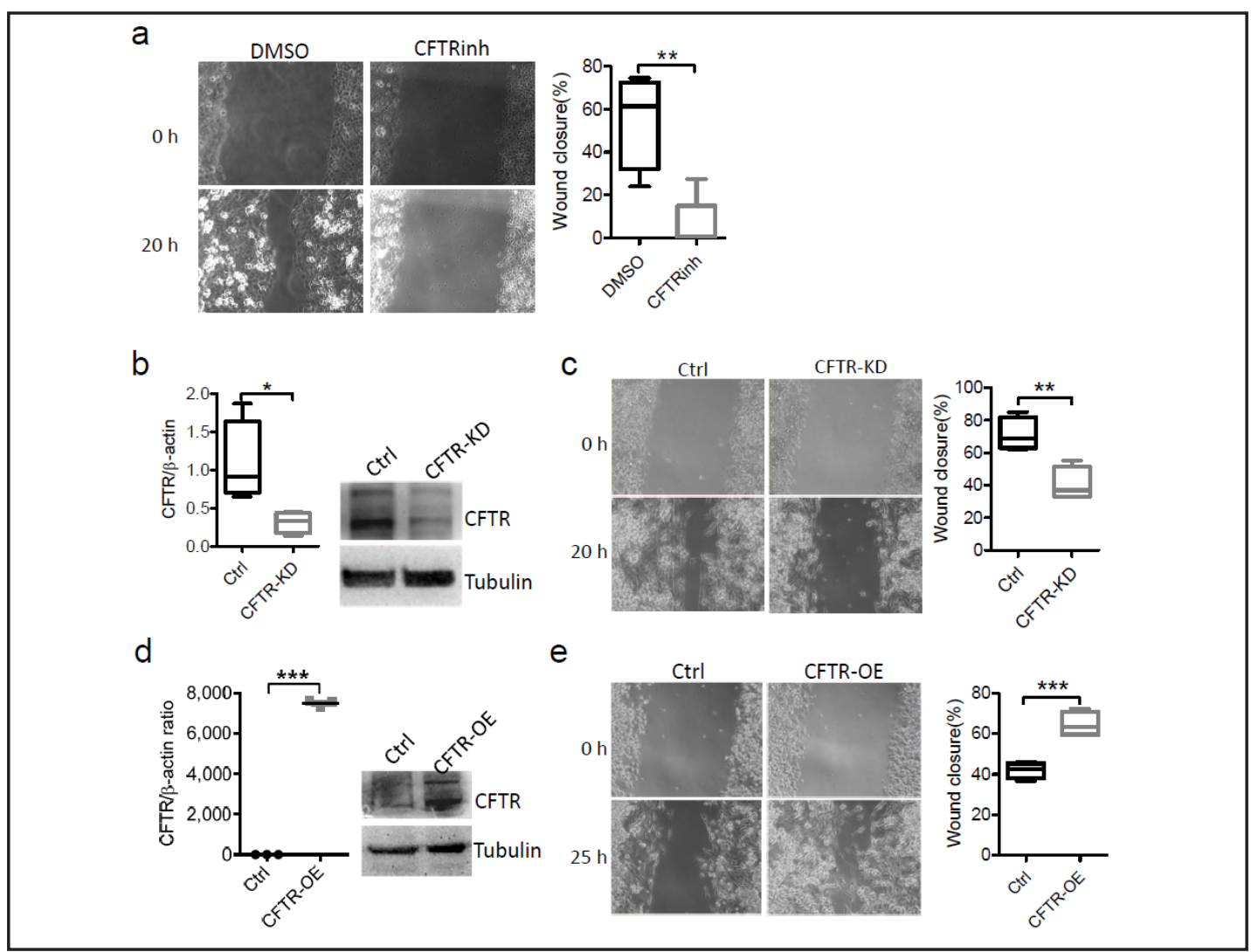

Fig. 2. CFTR promotes keratinocyte wound healing in vitro. a) Representative bright-field images and quantification of wound closure rate in HaCaT cells in presence of CFTR inhibitor-172 (CFTRinh, 10 $\mu$ M) or DMSO as control 0-20 hours after a line scratch wound was made. b-c) QRT-PCR and western blot analysis of CFTR expression (b) and assessment of wound healing 0-20 hours after scratch (c) in HaCaT cells transfected with CFTR-knockdown ribosomes (CFTR-KD) and empty control6/V5-His vectors as negative control (Ctrl). *: $\mathrm{P}<0.05$. **: $\mathrm{P}<0.01, \mathrm{n}=4-5$, t-test. d-e) QRT-PCR and western blot analysis of CFTR expression (d) and assessment of wound healing 0-25 hours after scratch (e) in cells transfected with empty pEGFPC3 vectors (Ctrl) or pEGFPC3 vector conjugated with human CFTR to overexpress CFTR (CFTR-OE). ***: P<0.001, n=34 , t-test.

\section{CFTR deficiency increases keratinocyte proliferation}

Delayed wound healing as observed both in vivo and in vitro might suggest altered keratinocyte proliferation. We therefore tested whether CFTR plays a role in keratinocytes proliferation. At day 5 post incisional wounding, significantly thicker epidermis and larger number of cells labeled with PCNA, a proliferation marker, in the epidermis near the wound were found in DF508 mice, as compared to the wildtypes (Fig. 4a). Moreover, in non-injured skin of DF508 mice, substantially thicker epidermis and more PCNA positive keratinocytes were also found, as compared to that of the wildtypes (Fig. 4b). Western blotting for PCNA in skin tissue homogenates also revealed higher expression level in DF508 as compared to the wildtypes (Fig. 4c). Consistently, knockdown of CFTR in HaCaT cells led to increased proliferation rate (Fig. 4d), whereas overexpression of CFTR resulted in decreased proliferation rate (Fig. 4e).

\section{CFTR promotes keratinocyte differentiation}

The observed delayed cutaneous wound healing with higher keratinocyte proliferation in DF508 mice or CFTR-knockdown HaCaT cells suggested that CFTR-defective keratinocytes may have differentiation defects in spite of higher proliferation. Indeed, significantly lower mRNA levels of keratinocyte differentiation markers including calmodulin 5 (CALML5), 
a
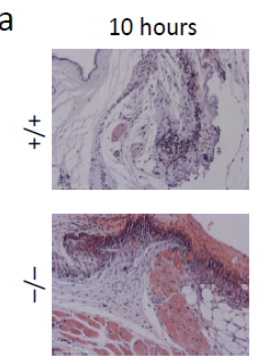

b

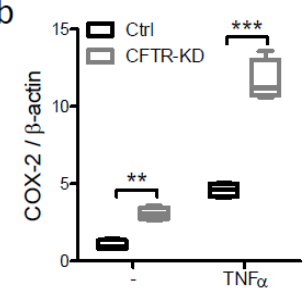

d

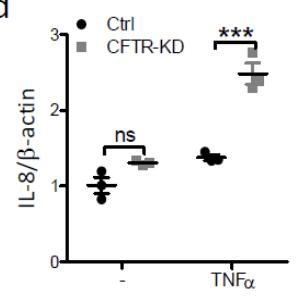

Day 3
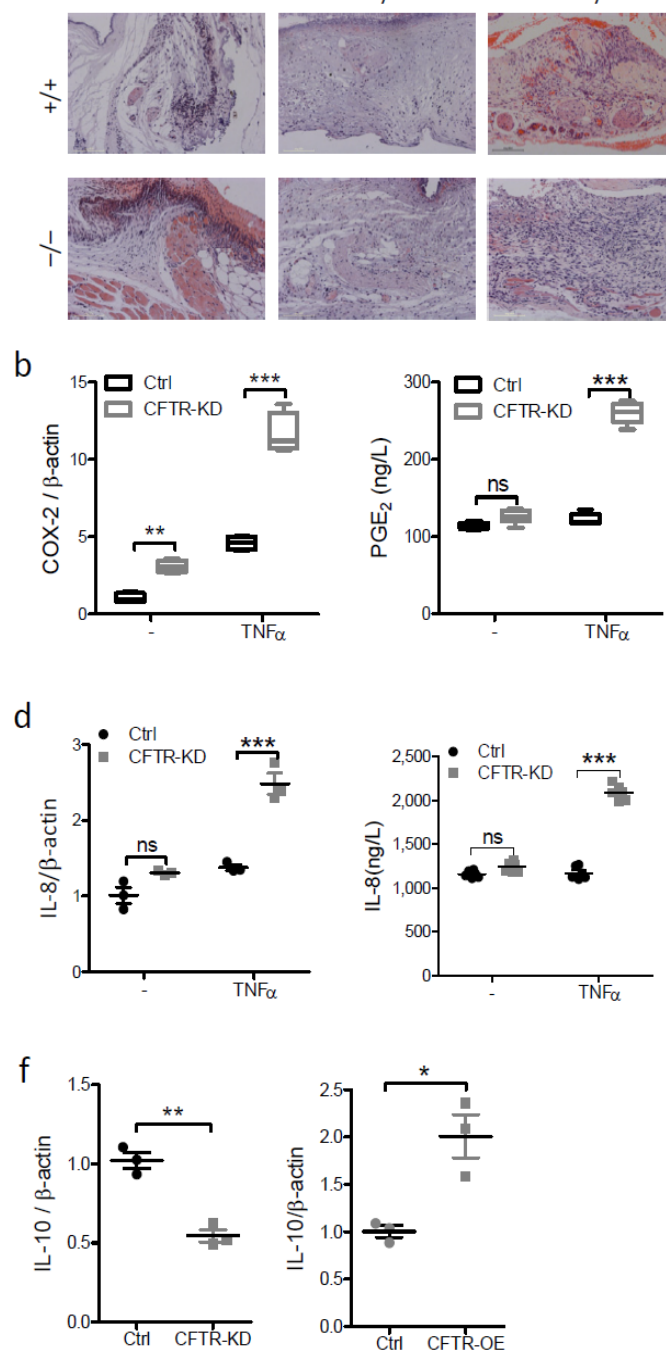

Day 5
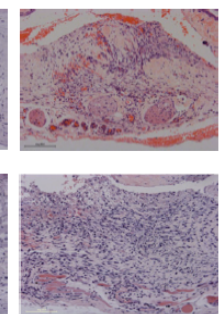

$\mathrm{TNF} \alpha$

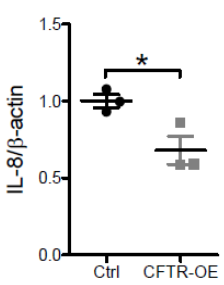

C
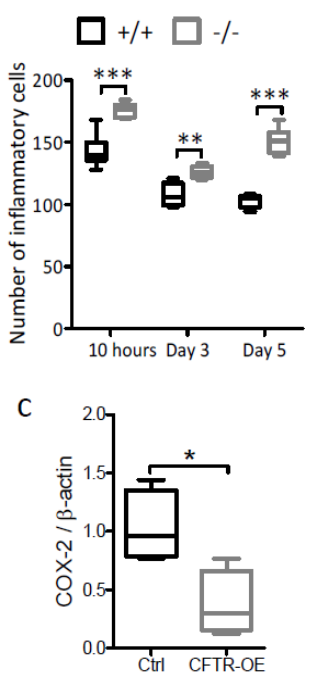

Fig. 3. CFTR deficiency enhances epidermal inflammation. a) H\&E staining and quantification of inflammatory cells in the wound bed of DF508 mice and wildtypes at 10 hours, day 3 and day5 post injury. Bar $=100 \mu \mathrm{m}$. **: $\mathrm{P}<0.01$, ***: $\mathrm{P}<0.001, \mathrm{n}=3$, Two-way ANOVA. b) QRT-PCR for COX-2 and ELISA for PGE ${ }_{2}$ in CFTR-KD or Ctrl cells with (+) or without (-) addition of TNF- $\alpha$. ${ }^{* *}$ : P<0.01, ${ }^{* * *}$ : $\mathrm{P}<0.001, \mathrm{n}=4-6$, Two-way ANOVA. c) QRT-PCR for COX-2 in CFTR-OE or Ctrl cells. *: P<0.05, n=3, t-test. d) QRT-PCR and ELISA for IL-8 CFTR-KD or Ctrl cells with (+) or without (-) addition of TNF $\alpha$. ns: non-significant. ***: $\mathrm{P}<0.001, \mathrm{n}=3$, Two-way ANOVA. e) QRTPCR for IL-8 in CFTR-OE or Ctrl cells. *: P<0.05, n=3, t-test. f) QRT-PCR for IL-10 in CFTR-KD, CFTR-OE or Ctrl cells. *: $\mathrm{P}<0.05$, **: $\mathrm{P}<0.01, \mathrm{n}=3$, t-test.

loricrin (LOR), filaggrin (FLG) and involucrin (IVL) were found in keratinocytes isolated from excisional wounds of DF508 mice as compared to those from the wildtypes (Fig. 5a). We next induced $\mathrm{HaCaT}$ cells differentiation by switching the cultures from $\mathrm{Ca}^{2+}$-free to $\mathrm{Ca}^{2+}(1 \mathrm{mM})$ contained medium. 48 hours after the $\mathrm{Ca}^{2+}$ switch, mRNA levels of CALML5, LOR, FLG and IVL were found significantly increased as compared to control cells, suggesting differentiation of HaCaT cells (Fig. 5b). In these $\mathrm{Ca}^{2+}$ treated cells, higher expression of CFTR was found as compared to control cells. Moreover, $\mathrm{Ca}^{2+}$ treated cells with CFTR knockdown exhibited significantly lower levels of LOR, CALML5, IVL and FLG as compared to control cells (Fig. 5c). Conversely, overexpression of CFTR increased the expression of these differentiation markers (Fig. 5d). 
Fig. 4. CFTR deficiency increase keratinocyte proliferation. a) Immunohistochemistry staining for PCNA in skin tissues from DF508 (-/-) and wild-type $(+/+)$ mice at day 5 after the incisional injury with epidermal thickness and number of PCNA positive cells quantified. Bars $=100 \mu \mathrm{m}$. ***: $\mathrm{P}<0.001$, $\mathrm{n}=4$, t-test. b) H\&E staining (left top, Bars $=200 \mu \mathrm{m}$ ) and immunohistochemistry staining for PCNA (left bottom, Bars $=100 \mu \mathrm{m}$ )with quantification (right) of epidermal thickness and number of PCNA-positive cells in uninjured skin of DF508 $(-/-)$ and wild-type $(+/+)$ mice. ${ }^{* * *}$ : $\mathrm{P}<0.001, \mathrm{n}=4$, t-test. c) Western blotting for PCNA in skin tissues from DF508 and wild-type mice. Protein extracts loaded in each lane were from skin tissues of four mice $(+/+$ or $-/-)$ at equal amount of weight. $n=4$. d-e) Cell proliferation assay in HaCaT cells treated with CFTR-KD (d) or CFTR-OE (e). ***: $\mathrm{P}<0.001, \mathrm{n}=5$, Two-way ANOVA.

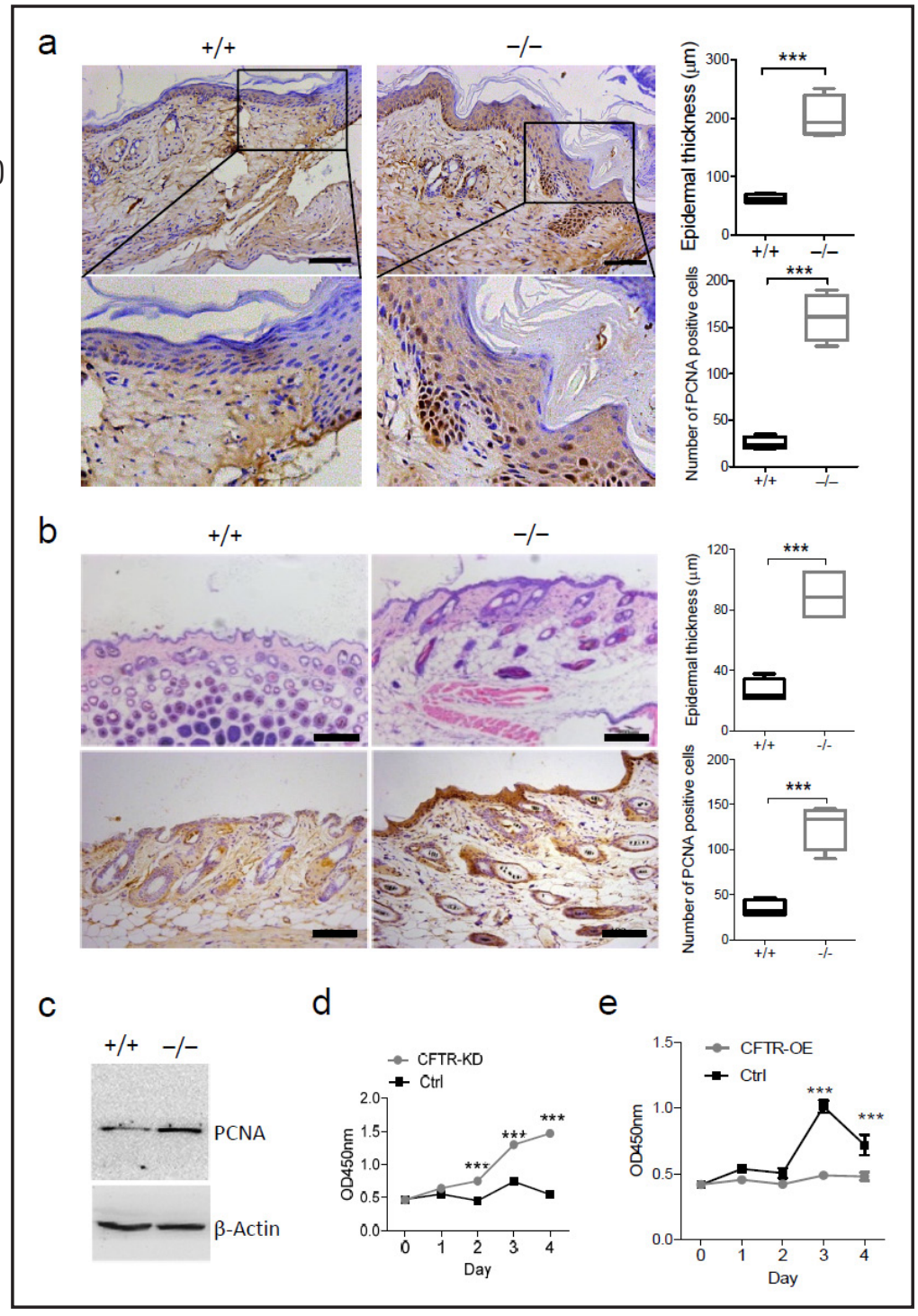

CFTR regulates epidermal inflammation, proliferation and differentiation via MAPK/NF$\kappa B$ pathway

The above results suggested the involvement of CFTR in inflammation, proliferation and differentiation of keratinocytes during cutaneous wound healing. We next tested possible role of MAPK or NF- $\mathrm{BB}$ in these processes, given their association with CFTRdeficiency reported in other cell types $[6,25,27,28]$. Results showed that in absence or presence of TNF $\alpha(10 \mu \mathrm{M})$, knockdown of CFTR in HaCaT cells induced phosphorylation of $\mathrm{I} \kappa \mathrm{B} \alpha$, indicating activation of NF- $\kappa \mathrm{B}$ pathway. Also, ERK and p38, two MAPKs, were found to be phosphorylated/activated in CFTR knockdown cells with or without TNF $\alpha$. We next examined the effects of inhibitors of NF- $\kappa$ B (Bay-11), ERK (PD98059) and p38 (SB203580) on inflammation, proliferation and differentiation in HaCaT cells in conjunction of CFTR knockdown. Results showed that upregulation of COX-2 in CFTR-knockdown cells was significantly attenuated by PD98059 $(20 \mu \mathrm{M})$-inhibition of ERK. Similarly, CFTR knockdowninduced IL-8 increase was significantly reduced by PD98059 $(20 \mu \mathrm{M})$ or SB203580 $(20 \mu \mathrm{M})$. More, the decrease in IL-10 as a result of CFTR knockdown was significantly reversed by either Bay-11 $(40 \mu \mathrm{M})$, PD98059 $(20 \mu \mathrm{M})$ or SB203580 $(20 \mu \mathrm{M})$ (Fig. 6b). HaCaT cells were next cultured in presence of these inhibitors and their proliferation was monitored daily after seeding. At day 4 and 5 post seeding, the enhanced proliferation in CFTR knock-down 
a
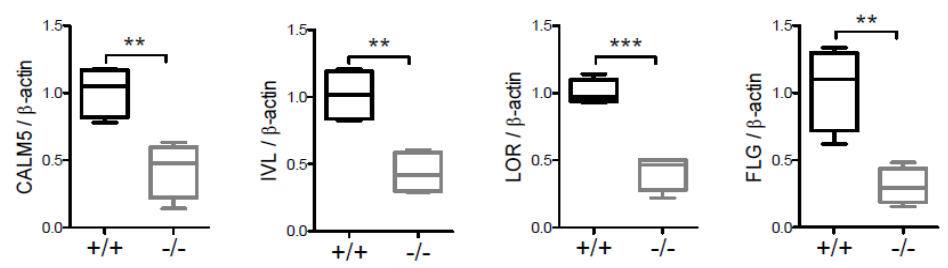

b
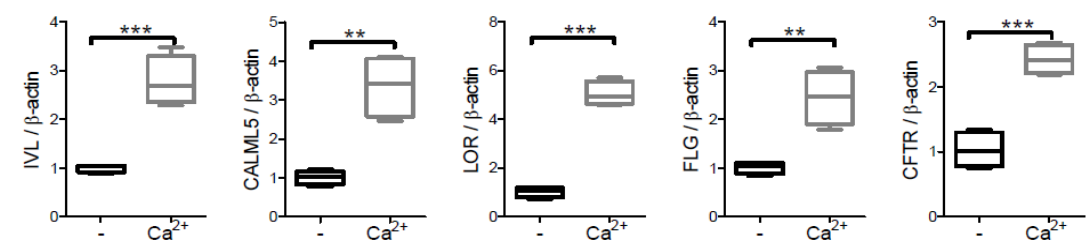

C
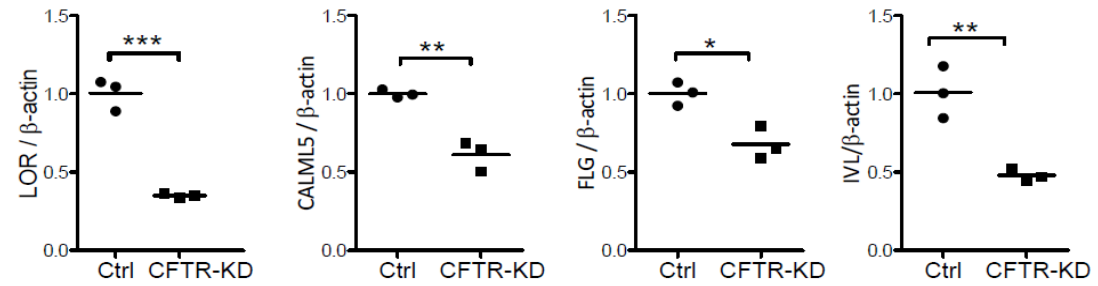

d
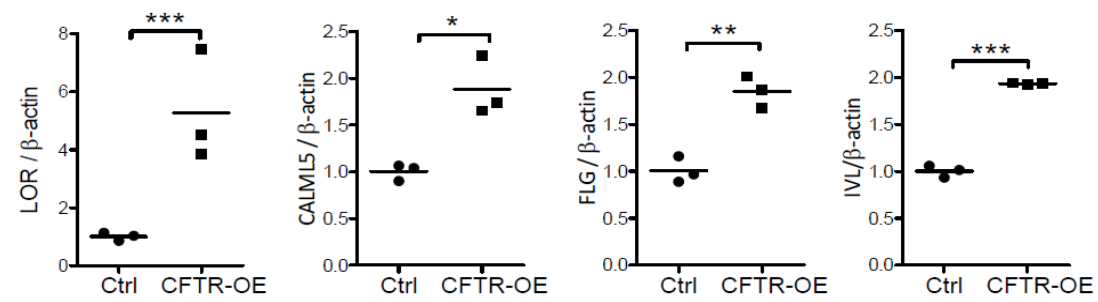

Fig. 5. CFTR promotes keratinocyte differentiation. a) QRT-PCR analysis of CALM5, LOR, FLG and IVL in isolated epidermal cells of DF508 and wild-type mice. ${ }^{* *}: \mathrm{P}<0.01,{ }^{* * *}: \mathrm{P}<0.001, \mathrm{n}=4, \mathrm{t}$ test. b) QRT-PCR analysis of LOR, CALM5, IVL, FLG and CFTR in HaCaT cells treated with or without $1 \mathrm{mM} \mathrm{Ca}^{2+}$. ** P<0.01; ***: $\mathrm{P}<0.001, \mathrm{n}=4$, t-test. c-d) QRT-PCR analysis of LOR, CALM5, IVL and FLG in HaCaT cells treated with CFTR-KD (d) or CFTR-OE (d) in presence of $1 \mathrm{mM} \mathrm{CaCl}_{2}$ to induce differentiation *: $\mathrm{P}<0.05$; **: $\mathrm{P}<0.01$; *** $\mathrm{P}<0.001, \mathrm{n}=3$, t-test.

cells was reduced by PD98059 $(20 \mu \mathrm{M})$ (Fig. 6c). Moreover, the downregulated IVL, FLG and CALML5 in CFTR-knockdown cells were all reversed by either Bay-11 $(40 \mu \mathrm{M})$, PD98059 $(20 \mu \mathrm{M})$ or SB203580 $(20 \mu \mathrm{M})$ (Fig. 6d).

\section{Discussion}

In summary, the present study has revealed a previously undefined role of CFTR in suppressing MAPK and NF- $\kappa$ B activation in epidermal keratinocytes during cutaneous injury to relieve inflammation, reduce keratinocyte proliferation and promote differentiation for a better wound healing.

Results from present in vivo and in vitro models clearly suggest a beneficial role of CFTR in cutaneous wound healing. Consistent with a previous study [31] showing retarded wound healing in DF508 mutant mice, the present study using a splint-excisional wound model, which better mimics cutaneous wound healing in humans, has confirmed that defects in 
Fig. 6. Involvement of $\mathrm{MAPK} / \mathrm{NF}-\kappa \mathrm{B}$ in CFTR-regulated keratinocyte function. a) Representative western blots and quantification for phosphorylated ERK (p-ERK), p38 (p-p38) and IкB $\alpha$ $(\mathrm{p}-\mathrm{I} \kappa \mathrm{B} \alpha)$ in $\mathrm{HaCaT}$ cells with or without CFTR-KD co-treated with or without TNF- $\alpha . \quad *$ : $\quad \mathrm{P}<0.05,{ }^{* *}$ : $\mathrm{P}<0.01, \mathrm{n}=3-5$, t-test. b-d) QRT-PCR analysis of COX-2, IL-8 and IL-10 (b), cell proliferation assay (c) and QRT-PCR analysis of IVL, FLG, CALM5 and LOR (d) in HaCaT cells with or without CFTR-KD co-treated with DMSO, Bay11 $(40 \mu \mathrm{M}), \quad$ PD98059 $(20 \mu \mathrm{M})$ or SB203580 $(20 \mu \mathrm{M})$ respectively. *: $\mathrm{P}<0.05, * *$ : $\mathrm{P}<0.01$; ***: $\mathrm{P}<0.001, \mathrm{n}=4$, One-way ANOVA.
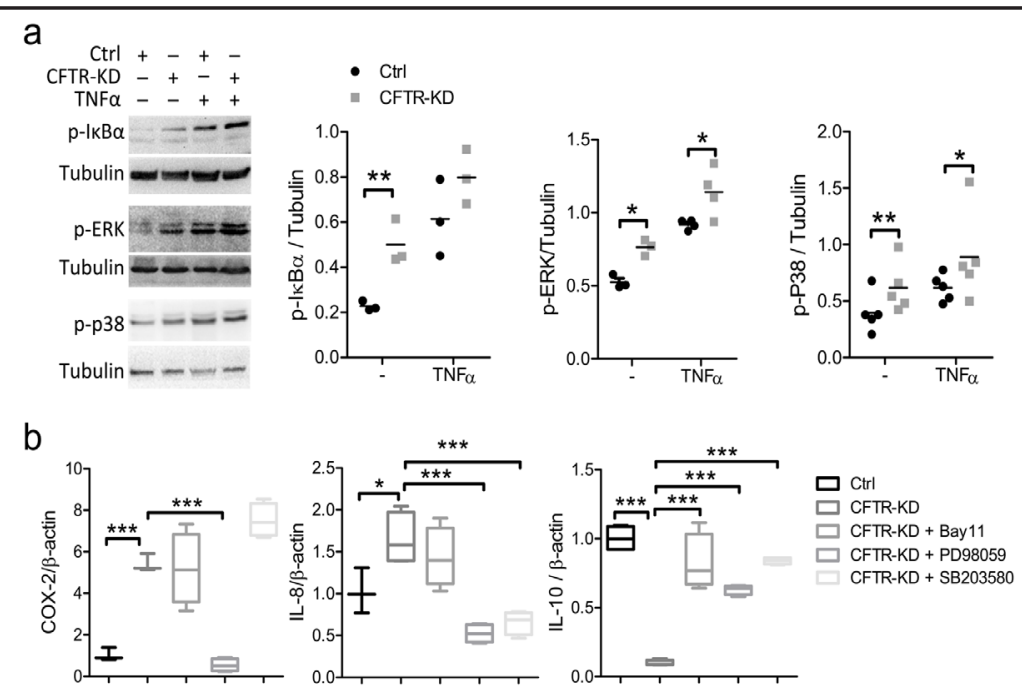

C

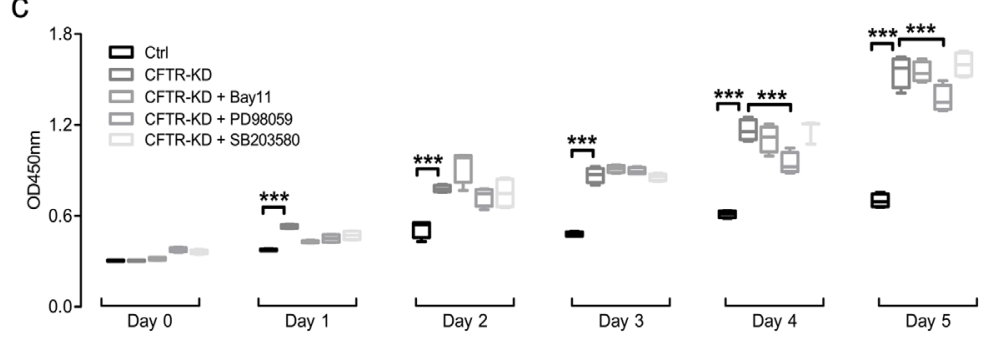

d
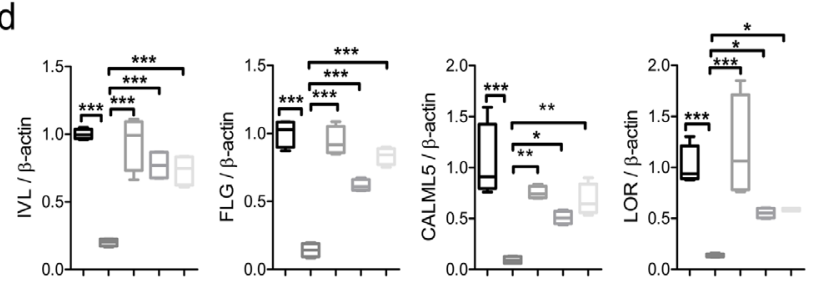

CFTR can result in impaired cutaneous wound healing. In addition, results from knockdown and overexpression of CFTR in HaCaT cells in conjunction of cell-scratch model have further confirmed an active role of keratinocyte CFTR in wound healing.

Multiple processes during cutaneous wound healing are suggested to be associated with CFTR as well as its regulated MAPKs and NF- $\kappa$ B. First, the injury-induced inflammation, as indicated by infiltration of inflammatory cells to the wound, increases in inflammatory cytokines (COX-2/PGE 2 IL-8) in keratinocytes or downregulation of anti-inflammatory cytokine (IL-10), is overwhelmed under CFTR mutant or knock-down conditions (Fig. 3). This is consistent with reported over-inflammation in other organ systems such as the airway in CF patients [6]. Such an over-inflammatory state in keratinocytes is reversed by CFTR overexpression or inhibition of MAPKs (i.e. ERK or p38) (Fig. 6b). It thus suggests a role of CFTR in suppression of MAPK in keratinocytes upon injuries to prevent detrimental over-inflammation for a better wound healing. Different from the airway cells [6], NF- $\kappa B$ in keratinocytes, though also activated by CFTR-deficiency and involved in IL-10 production (Fig. 6), does not seem to contribute to CFTR-deficiency-induced changes in COX-2/PGE ${ }_{2}$ or IL-8, mechanisms underlying which await further investigation.

Second, keratinocytes proliferation is substantially enhanced by CFTR-deficiency. This is evident by largely thickened epidermal layer, more PCNA-positive keratinocytes observed in DF508 mice, as well as a higher proliferation rate with CFTR knockdown and a lower rate with its overexpression in HaCaT cell cultures (Fig. 4). Interestingly, CFTR knockdown- 


\section{Cellular Physiology Cell Physiol Biochem 2016;39:2262-2274

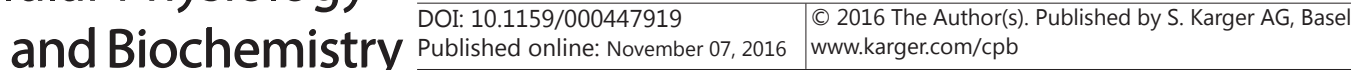 \\ Chen et al.: CFTR Suppresses MAPK/NF-kB in Cutaneous Wound Healing}

accelerated proliferation was reversed by inhibition of ERK, but not NFKB or p38, suggesting that activation of ERK, in addition to contributing to inflammation, also mediates the enhanced proliferation in CFTR-deficient keratinocytes (Fig 6c). However, using neonatal human keratinocytes (HEKn), Dong et.al showed comparable PCNA expression between CFTR-knockdown and control cells [31]. This might be due to different origins of HEKn and HaCaT, as HEKn cells are from neonatal human skin and HaCaT cells, from adult human skin [40]. Neonatal keratinocytes might have a very high proliferation rate that possibly compromises CFTR-induced effect. In adult DF508 mice and HaCaT cells, the present study suggests an active role of CFTR in suppressing keratinocyte proliferation.

Of note, the equilibrium between proliferating keratinocytes in the basal layer and their differentiation into spinous cells in the suprabasal layers of the epidermis is essential to the integrity and function of the epidermis. The alteration in keratinocyte proliferation induced by CFTR-deficiency may preclude keratinocyte differentiation or re-epithelialization required for the epidermis to be fully healed from injuries. Indeed, the differentiated keratinocytes markers including IVL, FLG, CAML5 and LOR are all downregulated in keratinocytes from DF508 mice or CFTR-knockdown HaCaT cells. This is consistent with previous studies that implicate CFTR in differentiation of different epithelial cell types, including keratinocytes $[24,30,31]$. Interestingly, the downregulation of these keratinocyte differentiation markers is largely reversed by inhibition of either NF- $\kappa B$, ERK or p38, suggesting NF- $\kappa B$ and MAPK pathways in mediating the effect of CFTR on keratinocyte differentiation during wound healing. Notably, it was reported that p38, in particular p38-delta, suppresses ERK activity in inhibiting keratinocyte differentiation $[13,41]$. However, in the present study, downregulation of differentiation markers in CFTR-knockdown cells was reversed by either PD98059, the ERK inhibitor, or SB203580, a selective inhibitor of p38-alpha and beta, suggesting inhibitory role of both p38 (alpha and beta) and ERK in keratinocytes differentiation under CFTRdeficient condition. Taken together, in addition to regulating tight-junction complex proteins required for epithelial differentiation as previously reported [24, 31], CFTR may additionally play a role in suppressing NF- $\kappa \mathrm{B}$ and MAPK pathways to promote re-epithelialization during cutaneous wound healing.

Similarly as observed presently in keratinocytes, the association of CFTR deficiency with MAPK or NF- $\kappa B$ activation has been reported in other organ-systems [6, 18, 25-28, 42] with various mechanisms proposed such as increased oxidative stress [27]. However, the exact mechanisms underlying how CFTR suppresses MAPK and NF- $\mathrm{BB}$ in keratinocytes during wound healing await further investigation.

Collectively, the present results have suggested a role of CFTR in keratinocytes in switching from inflammation/proliferation into differentiation/re-epithelialization to promote cutaneous wound healing. Of note, Psoriasis, an inflammatory skin disorder, is characterized by excessive inflammatory cytokines, keratinocyte hyper-proliferation as well as impaired differentiation[43], similarly as observed in CFTR-defective epidermis in the present study. Therefore, the present findings may suggest the potential of CFTR as a therapeutic target for such skin disease(s).

\section{Acknowledgements}

This work was supported by a grant from the State Key Laboratory of Trauma, Burns and Combined Injury of China (No. SKLZZ201230), the National Natural Science Foundation of China (No. 81201464, No. 81471460) and by a grant from the CPLA Scientific Research Fund (No. BWS11J039).

\section{Disclosure Statement}

The authors of this article declare that there are no conflicts of interest.

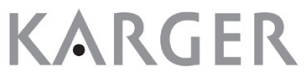




\section{Cellular Physiology Cell Physiol Biochem 2016;39:2262-2274 and Biochemistry \begin{tabular}{l|l} 
DOI: 10.1159/000447919 & $\begin{array}{l}\text { (c) } 2016 \text { The Author(s). Published by S. Karger AG, Basel } \\
\text { www.karger.com/cpb }\end{array}$
\end{tabular} \\ Chen et al:: CFTR Suppresses MAPK/NF-KB in Cutaneous Wound Healing}

\section{References}

1 Yamaguchi Y, Yoshikawa K: Cutaneous wound healing: an update. J Dermatol 2001;28:521-534.

2 Borena BM, Martens A, Broeckx SY, Meyer E, Chiers K, Duchateau L, Spaas JH: Regenerative Skin Wound Healing in Mammals: State-of-the-Art on Growth Factor and Stem Cell Based Treatments. Cell Physiol Biochem 2015;36:1-23.

3 Schreiber J, Efron Pa Fau - Park JE, Park Je Fau - Moldawer LL, Moldawer Ll Fau - Barbul A, Barbul A: Adenoviral gene transfer of an NF-kappaB super-repressor increases collagen deposition in rodent cutaneous wound healing. Surgery 2005;138:940-946.

4 Thuraisingam T, Xu YZ, Eadie K, Heravi M, Guiot MC, Greemberg R, Gaestel M, Radzioch D: MAPKAPK-2 signaling is critical for cutaneous wound healing. J Invest Dermatol 2010;130:278-286.

5 Heo SC, Jeon ES, Lee IH, Kim HS, Kim MB, Kim JH: Tumor necrosis factor-alpha-activated human adipose tissue-derived mesenchymal stem cells accelerate cutaneous wound healing through paracrine mechanisms. J Invest Dermatol 2011;131:1559-1567.

6 Chen J, Jiang XH, Chen H, Guo JH, Tsang LL, Yu MK, Xu WM, Chan HC: CFTR negatively regulates cyclooxygenase-2-PGE(2) positive feedback loop in inflammation. J Cell Physiol 2012;227:2759-2766.

$7 \quad$ Kyriakis JM, Avruch J: Mammalian MAPK signal transduction pathways activated by stress and inflammation: a 10-year update. Physiol Rev 2012;92:689-737.

8 Calautti E, Li J, Saoncella S, Brissette JL, Goetinck PF: Phosphoinositide 3-kinase signaling to Akt promotes keratinocyte differentiation versus death. J Biol Chem 2005;280:32856-32865.

9 Raman M, Chen W, Cobb MH: Differential regulation and properties of MAPKs. Oncogene 2007;26:31003112.

10 Hu Y, Baud V, Delhase M, Zhang P, Deerinck T, Ellisman M, Johnson R, Karin M: Abnormal morphogenesis but intact IKK activation in mice lacking the IKKalpha subunit of IkappaB kinase. Science 1999;284:316320.

11 Jonak C, Mildner M, Klosner G, Paulitschke V, Kunstfeld R, Pehamberger H, Tschachler E, Trautinger F: The hsp27kD heat shock protein and p38-MAPK signaling are required for regular epidermal differentiation. J Dermatol Sci 2011;61:32-37.

12 Hara T, Miyazaki M, Hakuno F, Takahashi S, Chida K: PKCeta promotes a proliferation to differentiation switch in keratinocytes via upregulation of p27Kip1 mRNA through suppression of JNK/c-Jun signaling under stress conditions. Cell Death Dis 2011;2:e157.

13 Popp T, Egea V, Kehe K, Steinritz D, Schmidt A, Jochum M, Ries C: Sulfur mustard induces differentiation in human primary keratinocytes: opposite roles of p38 and ERK1/2 MAPK. Toxicol Lett 2011;204:43-51.

14 Lopez-Pajares V, Yan K, Zarnegar BJ, Jameson KL, Khavari PA: Genetic pathways in disorders of epidermal differentiation. Trends Genet 2013;29:31-40.

15 Wilson JM, Collins FS: Cystic fibrosis. More from the modellers. Nature 1992;359:195-196.

16 Davis PB, Drumm M, Konstan MW: Cystic fibrosis. Am J Respir Crit Care Med 1996;154:1229-1256.

17 Sheppard DN, Carson MR, Ostedgaard LS, Denning GM, Welsh MJ: Expression of cystic fibrosis transmembrane conductance regulator in a model epithelium. Am J Physiol 1994;266:L405-413.

18 Vij N, Mazur S, Zeitlin PL: CFTR is a negative regulator of NFkappaB mediated innate immune response. PLoS One 2009;4:e4664.

19 Leigh MW, Kylander JE, Yankaskas JR, Boucher RC: Cell proliferation in bronchial epithelium and submucosal glands of cystic fibrosis patients. Am J Respir Cell Mol Biol 1995;12:605-612.

20 Chen H, Guo JH, Zhang XH, Chan HC: Defective CFTR-regulated granulosa cell proliferation in polycystic ovarian syndrome. Reproduction 2015;149:393-401.

21 Gallagher AM, Gottlieb RA: Proliferation, not apoptosis, alters epithelial cell migration in small intestine of CFTR null mice. Am J Physiol Gastrointest Liver Physiol 2001;281:G681-687.

22 Le Henaff C, Mansouri R, Modrowski D, Zarka M, Geoffroy V, Marty C, Tarantino N, Laplantine E, Marie PJ: Increased NF-kappaB Activity and Decreased Wnt/beta-Catenin Signaling Mediate Reduced Osteoblast Differentiation and Function in DeltaF508 Cystic Fibrosis Transmembrane Conductance Regulator (CFTR) Mice. J Biol Chem 2015;290:18009-18017.

23 Stalvey MS, Clines KL, Havasi V, McKibbin CR, Dunn LK, Chung WJ, Clines GA: Osteoblast CFTR inactivation reduces differentiation and osteoprotegerin expression in a mouse model of cystic fibrosis-related bone disease. PLoS One 2013;8:e80098. 


\section{Cellular Physiology Cell Physiol Biochem 2016;39:2262-2274 and Biochemistry Published online: November 07, 2016 \begin{tabular}{l|l} 
DOI: 10.1159/000447919 2016 The Author(s). Published by S. Karger AG, Basel \\
www.karger.com/cpb
\end{tabular} \\ Chen et al.: CFTR Suppresses MAPK/NF-KB in Cutaneous Wound Healing}

24 Ruan YC, Wang Y, Da Silva N, Kim B, Diao RY, Hill E, Brown D, Chan HC, Breton S: CFTR interacts with ZO-1 to regulate tight junction assembly and epithelial differentiation through the ZONAB pathway. J Cell Sci 2014;127:4396-4408.

25 Martel G, Roussel L, Rousseau S: The protein kinases TPL2 and EGFR contribute to ERK1/ERK2 hyperactivation in CFTRDeltaF508-expressing airway epithelial cells exposed to Pseudomonas aeruginosa. Biochem Biophys Res Commun 2013;pii:S0006-291X(13)01702-6.

26 Hunter MJ TK, Winter AK, Cassidy DM, Land S, Mehta A.: Expression of wildtype CFTR suppresses NFkappaB-driven inflammatory signalling. PLoS One 2010;5:e11598.

27 Berube J, Roussel L, Nattagh L, Rousseau S: Loss of cystic fibrosis transmembrane conductance regulator function enhances activation of p38 and ERK MAPKs, increasing interleukin-6 synthesis in airway epithelial cells exposed to Pseudomonas aeruginosa. J Biol Chem 2010;285:22299-22307.

28 Roque T, Boncoeur E, Saint-Criq V, Bonvin E, Clement A, Tabary O, Jacquot J: Proinflammatory effect of sodium 4-phenylbutyrate in deltaF508-cystic fibrosis transmembrane conductance regulator lung epithelial cells: involvement of extracellular signal-regulated protein kinase 1/2 and c-Jun-NH2-terminal kinase signaling. J Pharmacol Exp Ther 2008;326:949-956.

29 Dong ZW, Chen J, Ruan YC, Zhou T, Chen Y, Chen Y, Tsang LL, Chan HC, Peng YZ: CFTR-regulated MAPK/NFkappaB signaling in pulmonary inflammation in thermal inhalation injury. Sci Rep 2015;30:15946.

30 Sato F, Soos G, Link C, Sato K: Cystic fibrosis transport regulator and its mRNA are expressed in human epidermis. J Invest Dermatol 2002;119:1224-1230.

31 Dong J, Jiang X, Zhang X, Liu KS, Zhang J, Chen J, Yu MK, Tsang LL, Chung YW, Wang Y, Zhou WL, Chan HC: Dynamically Regulated CFTR Expression and Its Functional Role in Cutaneous Wound Healing. J Cell Physiol 2015;230:2049-2058.

32 Zhihui Yao YH, Gaoxing Luo, Jun Wu, Weifeng He: A biological membrane-based novel excisional woundsplinting model in mice. Burns \& Trauma 2014;2:196-200.

33 Deyrieux AF, Wilson VG: In vitro culture conditions to study keratinocyte differentiation using the HaCaT cell line. Cytotechnology 2007;54:77-83.

34 Pirrone A, Hager B, Fleckman P: Methods Mol Biol 2005;289:3-14.

35 Ansell DM, Campbell L, Thomason HA, Brass A, Hardman MJ: A statistical analysis of murine incisional and excisional acute wound models. Wound Repair Regen 2014;22:281-287.

36 Eming SA, Krieg T, Davidson JM: Inflammation in wound repair: molecular and cellular mechanisms. J Invest Dermatol 2007;127:514-525.

37 Hunter MJ, Treharne KJ, Winter AK, Cassidy DM, Land S, Mehta A: Expression of wild-type CFTR suppresses NF-kappaB-driven inflammatory signalling. PLoS One 2010;5:e11598.

38 Su X, Looney MR, Su HE, Lee JW, Song Y, Matthay MA: Role of CFTR expressed by neutrophils in modulating acute lung inflammation and injury in mice. Inflamm Res 2011;60:619-632.

39 LaDuca JR, Gaspari AA: Targeting tumor necrosis factor alpha. New drugs used to modulate inflammatory diseases. Dermatol Clin 2001;19:617-635.

40 Boukamp P, Petrussevska RT, Breitkreutz D, Hornung J, Markham A, Fusenig NE: Normal keratinization in a spontaneously immortalized aneuploid human keratinocyte cell line. J Cell Biol 1988;106:761-771.

41 Efimova T, Broome AM, Eckert RL: A regulatory role for p38 delta MAPK in keratinocyte differentiation. Evidence for p38 delta-ERK1/2 complex formation. J Biol Chem 2003;278:34277-34285.

42 Ntimbane T, Comte B, Mailhot G, Berthiaume Y, Poitout V, Prentki M, Rabasa-Lhoret R, Levy E: Cystic fibrosis-related diabetes: from CFTR dysfunction to oxidative stress. Clin Biochem Rev 2009;30:153-177.

43 Goldminz AM, Au SC, Kim N, Gottlieb AB, Lizzul PF: NF-kappaB: an essential transcription factor in psoriasis. J Dermatol Sci 2013;69:89-94. 